\title{
Sokal Score
}

National Cancer Institute

\section{Source}

National Cancer Institute. Sokal Score. NCI Thesaurus. Code C127875.

A method for assessing prognosis for patients with chronic myeloid leukemia. The numerical value is calculated at diagnosis from the percentage of blast cells in peripheral blood, the platelet count, the spleen size, and the age of the patient. 